RESEARCH

\title{
Liver findings in patients with Carney complex, germline PRKAR1A pathogenic variants, and link to cardiac myxomas
}

\author{
Amit Tirosh1,2, Ahmed Hamimi ${ }^{3}$, Fabio Faucz'1, Genya Aharon-Hananel2, Phaedon D Zavras', Belen Bonella5, \\ Adi Auerbach6, David Gillis7, Charalampos Lyssikatos', Elena Belyavskaya1, Constantine A Stratakis ${ }^{1, *}$ and \\ Ahmed M Gharib3,*
}

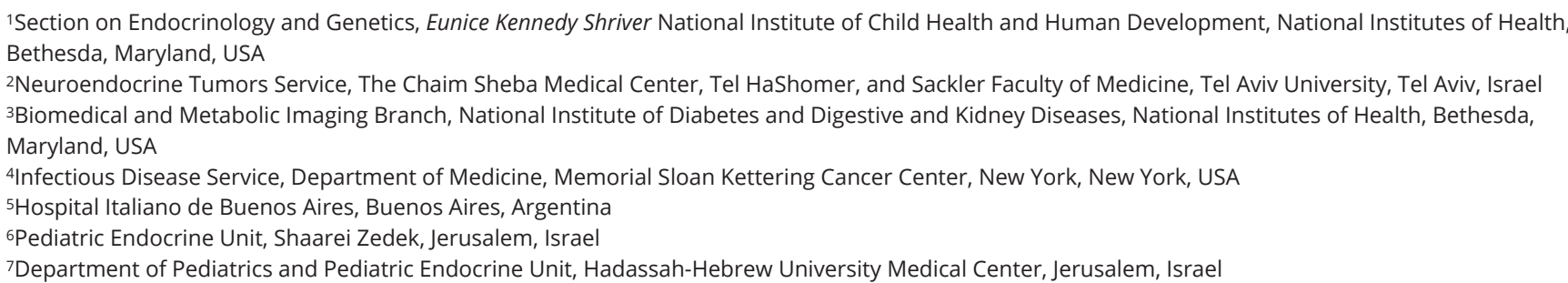

\begin{abstract}
This study aimed to evaluate liver involvement in patients with Carney complex (CNC) based on a large cohort and to analyze any germline PRKAR1A genotype-phenotype association of liver disease. The study included 83 patients with CNC, followed between 1995 and 2018 at a tertiary research center. We reviewed liver images, recorded types and number of lesions and analyzed per genotype: all patients were sequenced for the PRKAR1A gene. A total of $29 / 83$ patients $(24.0 \%)$ had liver radiological findings. Patients with liver lesion had a significantly higher rate of pathogenic variants detected in the PRKAR1A gene (72.4 vs $38.9 \%, P=0.005$, respectively). Patients with a pathogenic variant detected on germline PRKAR1A analysis had a higher risk for having a liver lesion compared with patients with wild-type (WT) PRKAR1A alleles (21/42 (50.0\%) vs 8/41 (19.5\%), respectively, $P=0.004)$. Among patients with liver lesions, those with a nonsense PRKAR1A pathogenic-variant had more liver lesions (7/7) than among those with other pathogenicvariant types $(8 / 22, P=0.001)$. In multivariable analysis, detection of liver lesion(s) was associated with an odds ratio of 5.2 for cardiac myxomas ( $95 \% \mathrm{Cl} 1.55-17.49, P=0.008)$. In conclusion, patients with CNC, particularly with a PRKAR1A pathogenic variant, have a higher rate of liver lesions. Additionally, liver lesions are associated with a high risk for cardiac myxomas in this population.
\end{abstract}




\section{Introduction}

Carney complex (CNC) is a rare multiple neoplasia syndrome, that is either inherited as an autosomal dominant trait, or occurs sporadically as a result of a de novo defect (Correa et al. 2015). CNC is mostly caused by a germline pathogenic variant in the PRKAR1A gene (OMIM 188830), which encodes the regulatory subunit type I alpha of protein kinase A (Kirschner et al. 2000).

$\mathrm{CNC}$ is a multi-organ syndrome and it most commonly manifests with spotty skin pigmentation, cutaneous, cardiac and breast myxomas, and primary pigmented nodular adrenocortical disease (PPNAD) associated with an atypical form of Cushing syndrome (CS) (Stratakis 2016). However, patients with CNC may also have other endocrine neoplasms including growth hormone- and/or prolactin-secreting pituitary adenoma(s), thyroid neoplasms, large-cell calcifying Sertoli cell tumors in the testis and ovarian cysts and carcinomas (Courcoutsakis et al. 2013).

We have recently reported a higher frequency of liver manifestations in pediatric patients with CNC, in association with failure to thrive (Tirosh et al. 2018). Liver involvement in CNC has been reported previously in case reports and small case series (Terracciano et al. 2004, Veugelers et al. 2004, Gennari et al. 2008), with some support from animal models, which developed liver lesions in association with abnormal protein kinase A activity (Veugelers et al. 2004).

However, until today liver involvement has not been validated in a large cohort of patients with CNC. Hence, in the current study we performed an imaging-based screening for liver manifestations in patients with CNC. We then analyzed our data that were collected without prior knowledge of the genetics, as per the genotype. Clearly, patients with CNC due to PRKAR1A pathogenic variant had more liver lesions.

\section{Patients and methods}

This is a retrospective study of 83 patients with $\mathrm{CNC}$, followed between 1995 and 2018 at the Eunice Kennedy Shriver National Institute of Child Health and Human Development (NICHD). One patient was diagnosed, treated and followed at Hadassah-Hebrew University Medical Center and genetic diagnosis was confirmed at the NIH. The NICHD Institutional Review Board has approved the study of patients with CNC (protocol 95-CH-0059); the parents of the patients signed the proper consent forms for clinical and genetic investigations related to the diagnosis of their child.
The diagnosis of CNC was made by using previously published diagnostic criteria (Correa et al. 2015). Genetic sequencing for PRKAR1A defects was obtained in all patients as was previously described (Kirschner et al. 2000). The patients' medical files were reviewed, and data were retrieved on demographics, medical and family history, physical findings, biochemical evaluation results, adrenal and liver imaging and treatment. Analysis of the imaging files and diagnoses were recorded without knowledge of the genetic testing results.

\section{Imaging techniques}

Examinations of the liver were done using commercially available high MRI and/or computerized tomography (CT) scanners for all subjects. The routine liver imaging examinations were evaluated by two radiologists in consensus who were blinded to genetic results, as above. Both radiologists had at least 15 years of experience in body and liver imaging. Standard diagnostic criteria and methods were used (Sahani \& Kalva 2004, Jang et al. 2009). Briefly, cysts are defined as hypodense well-defined lesion(s) on CT with no enhancement in any of the phases of enhancement. On MRI, cysts are T1 hypointense (dark) and T2 hyperintense (bright) with no mural enhancement noted after IV contrast administration. Adenoma is initially hypodense in CT with intense enhancement in arterial phase and wash-out in delayed phases. On MRI the lesion is T1 hypointense and T2 iso- to mildly hyperintense with enhancement pattern similar to that in CT. Hemangioma is hypodense in CT with initial nodular enhancement in early phases and gradual fill-in in portovenous and delayed phases. On MRI, hemangioma is T2 hyperintense and remain hyperintense in heavy T2WI with enhancement pattern parallel to that described in CT.

\section{Genetic analysis}

DNA was extracted from peripheral blood leukocytes using the Quick-DNA ${ }^{\mathrm{TM}}$ Universal Kit, according to manufacturer protocols (Zymo). The complete PRKAR1A coding sequence of the blood samples was analyzed by bidirectional Sanger sequencing, as we have previously described.

\section{Statistical analysis}

The analysis was performed on R statistics (version 3.4.4) via $\mathrm{R}$ studio (version 1.0.153). Data were described with simple descriptive statistics. Continuous variables are 


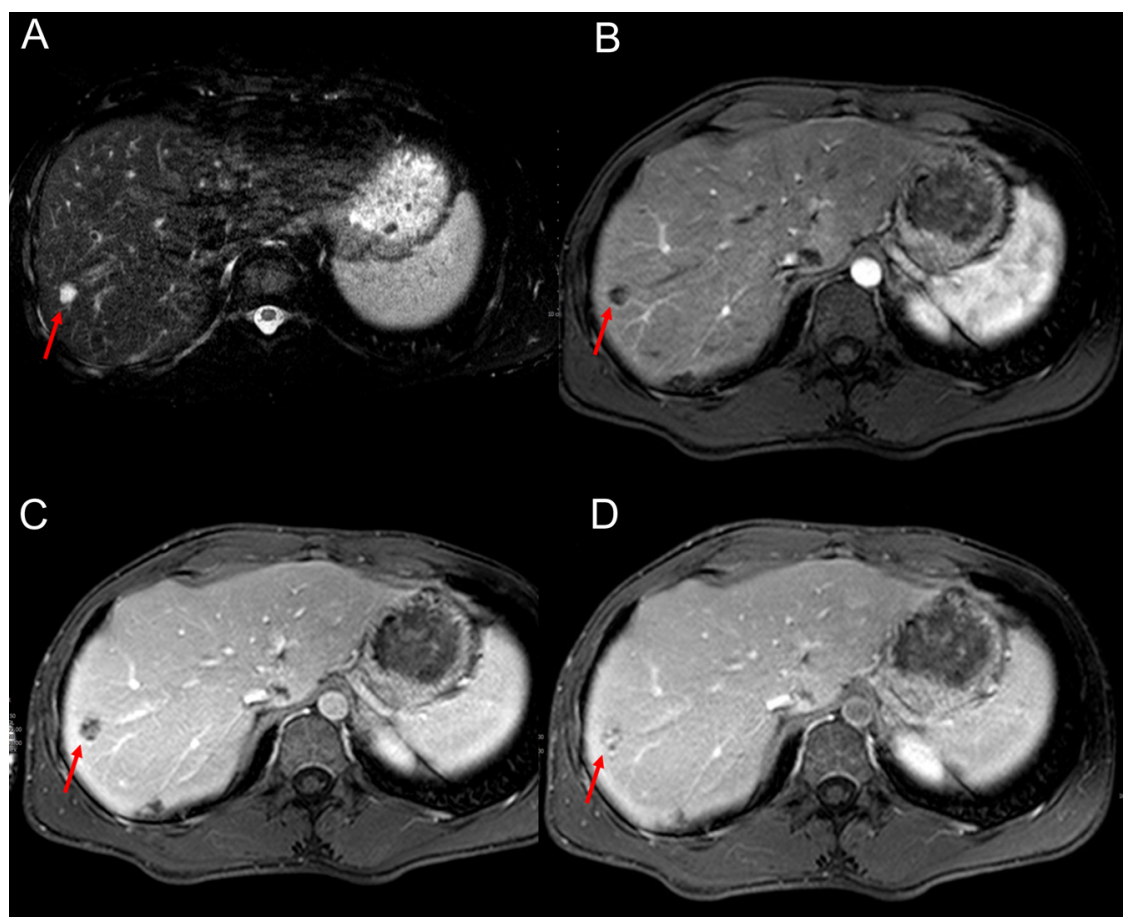

\section{Figure 1}

Magnetic resonance imaging of the liver of a 28-year-old male with Carney complex showing hemangioma. T2-weighted image showing bright lesion in right lobe of the liver (A). T1-weighted image with contrast in arterial phase showing no enhancement (B) with subsequent filling-in on porto-venous phase (C) and delayed venous phase (D). This pattern is consistent with hemangioma. A full colour version of this figure is available at https://doi.org/10.1530/ERC-19-0517.

presented as mean \pm s.D. and were compared using the Student's $t$-test, while parametric variables were compared using the chi-square test, unless otherwise indicated. Multivariable analysis was performed using logistic regression, including age (categorized to $\leq 40$ years and $>40$ years), gender, diagnosis with PPNAD and detection of liver lesion. Odds ratio (OR), 95\% CI and adjusted $P$ value are presented for each variable in the model. Statistical significance was set at two tailed $P$ value $<0.05$.

\section{Results}

The current analysis included 83 patients with CNC, with an age at last follow-up of $39.3 \pm 17.3$ years (range 9-75), including 59 females (71.1\%). Of 83 patients included in the current analysis, 42 had germline pathogenic variant detected in the PRKAR1A gene, including 15 frameshift mutations, 14 nonsense mutations, 8 splice site variants, 3 missense mutations and 2 unknown types of pathogenic variants.

A total of 29 patients $(24.0 \%)$ had liver radiological findings: 17 cysts, 3 cysts with other enhancing lesions, 2 hemangiomas (Fig. 1) and 6 undefined hepatic lesions. Patients with liver lesions had a significantly higher rate of pathogenic variants detected in the PRKAR1A gene (72.4 vs $38.9 \%, P=0.005$, respectively) and were older at last evaluation $(43.6 \pm 6.0$ vs $34.9 \pm 17.5$ years, respectively,
$P=0.02)$. The patients' characteristics, compared by presence/absence of liver lesion, are detailed in Table 1.

Patients with pathogenic variants detected on germline PRKAR1A analysis had a higher risk for having a liver lesion compared with patients with wild-type (WT) PRKAR1A alleles (21/42 (50.0\%) vs 8/41 (19.5\%), respectively, $P=0.004)$. Among patients with any liver lesion, patients harboring pathogenic variants in the PRKAR1A gene had higher rate of multiple liver lesions compared with patients with WT PRKAR1A allele, with borderline statistical significance (13/21 (61.9\%) vs $2 / 8$ (25.0\%), respectively, $P=0.08)$.

No difference was found in the risk to develop liver lesions between patients with different pathogenic variant types $(P=1.0)$; however, among patients with a liver lesion, all patients with nonsense PRKAR1A pathogenic variant had multiple liver lesions $(7 / 7,100 \%)$ vs $8 / 22$ (36.4\%) for patients with any other pathogenic variant types $(P=0.001)$.

To better assess the clinical implication of the liver lesion detection in CNC, we reviewed all the pathology reports of patients with reported liver lesions in our cohort. We found three liver tissue pathology reports: one patient underwent biopsy for a liver mass that was diagnosed as focal nodular hyperplasia, a second patient underwent adrenalectomy, with resection of normal liver tissue and the last had metastatic breast cancer with liver metastases, with normal background liver parenchyma. 
Table 1 Patients characteristics, compared by presence/absence of liver lesion on abdominal imaging.

\begin{tabular}{l}
\hline \\
\hline Female, $n(\%)$ \\
Age (years) \\
Imaging type (CT/MRI/US, $n(\%))$ \\
Number of lesion $n(\%)$ \\
$\quad$ Single lesion \\
Multiple lesions \\
Lesion characteristics (cyst/adenoma/hemangioma) \\
PPNAD (yes/no/unknown, $n$ (\%)) \\
Cardiac myxoma (yes/no/unknown, $n$ (\%)) \\
Germline pathogenic variant in PRKAR1A detected $n$ (\%) \\
Serum alkaline phosphatase level (IU/L) \\
Serum ALT level (IU/L) \\
Serum AST level (IU/L) \\
Serum total bilirubin level (mg/dL)
\end{tabular}

\begin{tabular}{c} 
Liver lesion \\
\hline Not detected $(n=54)$ \\
\hline $41 / 54(75.9)$ \\
$34.9 \pm 17.5$ \\
$9 / 42 / 3(16.7 / 77.8 / 5.6)$ \\
$\mathrm{NA}$ \\
$\mathrm{NA}$ \\
$30 / 8 / 16(55.6 / 14.8 / 29.6)$ \\
$14 / 24 / 16(25.9 / 44.4 / 29.6)$ \\
$21 / 54(38.9)$ \\
$95.2 \pm 70.3$ \\
$25.4 \pm 18.6$ \\
$21.1 \pm 7.8$ \\
$0.51 \pm 0.30$
\end{tabular}

\begin{tabular}{c} 
sion on imaging \\
Detected $(n=29)$ \\
\hline $18 / 29(62.1)$ \\
$43.6 \pm 6.0$ \\
$4 / 25 / 0(13.8 / 86.2)$ \\
\\
$14 / 29(48.3 \%)$ \\
$15 / 29(51.7 \%)$ \\
$19 / 5 / 5(65.52 / 17.24 / 17.24)$ \\
$16 / 7 / 6(55.2 / 24.1 / 20.7)$ \\
$9 / 13 / 7(31.0 / 44.8 / 24.1)$ \\
$21 / 29(72.4)$ \\
$81.9 \pm 55.2$ \\
$34.7 \pm 31.6$ \\
$24.0 \pm 15.4$ \\
$0.51 \pm 0.26$
\end{tabular}

\begin{tabular}{l}
\hline P value \\
\hline 0.2 \\
0.02 \\
0.4 \\
NA \\
NA \\
0.5 \\
0.8 \\
0.005 \\
0.4 \\
0.2 \\
0.3 \\
1.0
\end{tabular}

IU/L, international units/liter; NA, not applicable; PPNAD, primary pigmented nodular adrenocortical disease; US, ultrasound.

Since cortisol and growth hormone over-secretion have systematic manifestations, we screened for liver lesions among patients with non-CNC related acromegaly and Cushing syndrome. Among 95 patients with hypercortisolemia, 15 (23.5\%) had focal liver lesion (7 liver cysts, 3 hemangiomas, 1 adenoma and 4 lesions too small to characterize). The rate of liver lesions was significantly higher among patients with CNC in general vs non-CNC hypercortisolemia ( 34.9 vs $15.8 \%, P=0.003$ ), and especially compared to patients with $\mathrm{CNC}$ with pathogenic variant in PRKAR1A (50.0 vs $15.8 \%, P<0.001$ ). Among 10 patients with acromegaly, none had focal liver abnormality, lower than patients with CNC (15.8\% 0\%, Fisher's exact, $P=0.003$ ).

In order to assess the potential prognostic utility of liver lesion detection on other manifestations of $\mathrm{CNC}$, we performed logistic regression, controlling for demographic and clinical covariates. In the multivariable analysis, detection of any liver lesion(s) in patients with CNC was associated high risk for cardiac myxomas (odds ratio (OR) $5.21,95 \%$ CI $1.55-17.49, P=0.008$ ), in contrast to female gender (OR 1.84, 95\% CI 0.52-6.58, $P=0.35$ ), older age ( $>40$ years, OR 1.1, 0.3-3.3, $P=0.93$ ) and diagnosis with PPNAD (OR 0.32, 95\% CI 0.08-1.35, $P=0.12$ ).

\section{Discussion}

In the current study we report for the first time a high prevalence of liver lesions in a large cohort of patients with $\mathrm{CNC}$, based on a prospective natural history study with genetic analysis. We found a high rate of cystic lesions, together with liver nodules and hemangiomas, which were most common among patients with a pathogenic variant detected in the PRKAR1A gene. Moreover, patients with a detected pathogenic variant in the germline DNA, had increased risk for multiple liver lesions. Specifically, patients with nonsense PRKAR1A pathogenic variants had higher risk for multiple liver lesions compared with any other pathogenic variant type.

The prevalence of hepatic cysts and hemangiomas in the general population is debatable due to the high discrepancy in the published literature. Nevertheless, both cysts and other hepatic lesions are increasingly found as a mere coincidence on abdominal imaging techniques, such as ultrasonography CT and MRI (Lantinga et al. 2013). The prevalence of simple hepatic cysts is estimated by $15-18 \%$ in the United States (Lantinga et al. 2013) and the prevalence of hepatic hemangiomas is estimated as $0.4-10 \%$ based on radiological findings and autopsy series (Brouwers et al. 1997, Choi \& Nguyen 2005, Mocchegiani et al. 2016).

When compared to the general population, the rates of liver cysts and hemangiomas is not exceeding 20\%, whereas in our analysis, the rate of liver lesions (mainly cysts and hemangiomas) in patients with a detected PRKAR1A pathogenic variant was 50\% and most of these patients (61\%) had multiple lesions. Furthermore, multiple liver lesions are rare in the general population and are usually associated with either polycystic kidney disease, affecting up to $0.2 \%$ of the general population (Lavö et al. 1990), or isolated polycystic liver disease (has prevalence of less than 0.01\%) (Qian 2010).

Patients with multiple liver lesions in the non-CNC context are usually asymptomatic. However, a small fraction of these patients develop acute liver cyst-related
(C) 2020 Society for Endocrinology Published by Bioscientifica Ltd. Printed in Great Britain 
complications and/or massive cystic liver enlargement, causing morbidity and mortality (Qian 2010). Currently, the management of symptomatic patients is centered on palliating symptoms and treating complications; however, new therapies are under investigation (Masyuk et al. 2017). The absence of pathological finding in liver pathology reports in patients with CNC in our cohort suggests low clinical implication for this manifestation per se. However, with the high risk found for cardiac myxomas among patients with liver lesions in the multivariable analysis, detecting such lesion should alert the clinician to survey for cardiac myxomas more closely.

Our findings raise a question regarding screening strategies for liver lesions in patients with CNC and in particular in patients with a detected PRKAR1A pathogenic variant. With that being said, the clinical significance of multiple liver cysts in CNC is not known and further investigation is needed.

This analysis is based on the largest CNC patient cohort worldwide. Nevertheless, it has its limitations, including the retrospective methodology and the crosssectional analysis, with their known drawbacks. In addition, long-term follow-up on the complications of liver disease is required to establish the benefit from screening.

In conclusion, patients with CNC have a high rate of liver lesions, with higher rates in the patients' subgroups with a PRKAR1A pathogenic variant detected, and specifically in those harboring a nonsense pathogenic variant. Although liver lesions by themselves might not indicate follow-up or intervention based on our data, a closer surveillance for cardiac myxomas development may be indicated among patients in which liver lesions are detected.

\section{Declaration of interest}

The authors declare that there is no conflict of interest that could be perceived as prejudicing the impartiality of the research reported.

\section{Funding}

This work did not receive any specific grant from any funding agency in the public, commercial, or not-for-profit sector.

\section{Ethical approva}

The study was approved by the institutional Ethical Committee, and informed consent was obtained from all individual participants included in the study.

\section{Author contribution statement}

Manuscript writing: A T, G A H. Data collection and analysis: A T, G A H, P D Z, B B, A A, D G, C L, E B. Critical review of the manuscript: all authors. Tissue collection and genetic analysis (sequencing): $F$ F, C L, E B. MRI review and analysis: A H, A M G. Study design conception: C A S, A M G. C A S and A M G: authors contributed equally.

\section{References}

Brouwers MA, Peeters PM, de Jong KP, Haagsma EB, Klompmaker IJ, Bijleveld CM, Zwaveling JH \& Slooff MJ 1997 Surgical treatment of giant haemangioma of the liver. British Journal of Surgery 84 314-316. (https://doi.org/10.1046/j.1365-2168.1997.02534.x)

Choi BY \& Nguyen MH 2005 The diagnosis and management of benign hepatic tumors. Journal of Clinical Gastroenterology 39 401-412.

Correa R, Salpea P \& Stratakis CA 2015 Carney complex: an update. European Journal of Endocrinology 173 M85-M97. (https://doi. org/10.1530/EJE-15-0209)

Courcoutsakis NA, Tatsi C, Patronas NJ, Lee CC, Prassopoulos PK \& Stratakis CA 2013 The complex of myxomas, spotty skin pigmentation and endocrine overactivity (Carney complex): imaging findings with clinical and pathological correlation. Insights into Imaging 4 119-133. (https://doi.org/10.1007/s13244012-0208-6)

Gennari M, Stratakis CA, Hovarth A, Pirazzoli P \& Cicognani A 2008 A novel PRKAR1A mutation associated with hepatocellular carcinoma in a young patient and a variable Carney complex phenotype in affected subjects in older generations. Clinical Endocrinology 69 751-755. (https://doi.org/10.1111/j.1365-2265.2008.03286.x)

Jang HJ, Yu H \& Kim TK 2009 Imaging of focal liver lesions. Seminars in Roentgenology 44 266-282. (https://doi.org/10.1053/j. ro.2009.05.008)

Kirschner LS, Carney JA, Pack SD, Taymans SE, Giatzakis C, Cho YS Cho-Chung YS \& Stratakis CA 2000 Mutations of the gene encoding the protein kinase A type I-alpha regulatory subunit in patients with the Carney complex. Nature Genetics 26 89-92. (https://doi. org/10.1038/79238)

Lantinga MA, Gevers TJG \& Drenth JPH 2013 Evaluation of hepatic cystic lesions. World Journal of Gastroenterology 19 3543-3554. (https://doi.org/10.3748/wjg.v19.i23.3543)

Lavö B, Knutson L, Lööf L, Odlind B \& Hällgren R 1990 Signs of increased leakage over the jejunal mucosa during gliadin challenge of patients with coeliac disease. Gut 31 153-157. (https://doi. org/10.1136/gut.31.2.153)

Masyuk TV, Masyuk AI \& LaRusso NF 2017 Therapeutic targets in polycystic liver disease. Current Drug Targets 18 950-957. (https://doi. org/10.2174/1389450116666150427161743)

Mocchegiani F, Vincenzi P, Coletta M, Agostini A, Marzioni M, Baroni GS, Giovagnoni A, Guerrieri M, Marmorale C, Risaliti A, et al. 2016 Prevalence and clinical outcome of hepatic haemangioma with specific reference to the risk of rupture: a large retrospective crosssectional study. Digestive and Liver Disease 48 309-314. (https://doi. org/10.1016/j.dld.2015.09.016)

Qian Q 2010 Isolated polycystic liver disease. Advances in Chronic Kidney Disease 17 181-189. (https://doi.org/10.1053/j. ackd.2009.12.005)

Sahani DV \& Kalva SP 2004 Imaging the liver. Oncologist 9 385-397. (https://doi.org/10.1634/theoncologist.9-4-385)

Stratakis CA 2016 Carney complex: a familial lentiginosis predisposing to a variety of tumors. Reviews in Endocrine and Metabolic Disorders 17 367-371. (https://doi.org/10.1007/s11154-016-9400-1) https://erc.bioscientifica.com https://doi.org/10.1530/ERC-19-0517 (c) 2020 Society for Endocrinology Published by Bioscientifica Ltd. Printed in Great Britain 
Terracciano LM, Tornillo L, Avoledo P, Von Schweinitz D, Kühne T \& Bruder E 2004 Fibrolamellar hepatocellular carcinoma occurring 5 years after hepatocellular adenoma in a 14-year-old girl: a case report with comparative genomic hybridization analysis. Archives of Pathology and Laboratory Medicine 128 222-226. (https://doi. org/10.1043/1543-2165(2004)128<222:FHCOYA>2.0.CO;2)

Tirosh A, Auerbach A, Bonella B, Zavras PD, Belyavskaya E, Lyssikatos C, Meir K, Weiss R, Daum H, Lodish MB, et al. 2018 Failure to thrive in the context of Carney complex. Hormone Research in Paediatrics 89 38-46. (https://doi.org/10.1159/000484690)

Veugelers M, Wilkes D, Burton K, McDermott DA, Song Y,

Goldstein MM, La Perle K, Vaughan CJ, O'Hagan A, Bennett KR, et al. 2004 Comparative PRKAR1A genotype-phenotype analyses in humans with Carney complex and prkar1a haploinsufficient mice. PNAS 101 14222-14227. (https://doi.org/10.1073/ pnas.0405535101)

Received in final form 24 March 2020

Accepted 30 March 2020

Accepted Manuscript published online 17 April 2020
(C) 2020 Society for Endocrinology Published by Bioscientifica Ltd. Printed in Great Britain 